\title{
A FINITENESS CONDITION FOR LOCALLY COMPACT ABELIAN GROUPS
}

\author{
L. C. GROVE and L. J. LARDY
}

(Received 7 July 1969)

Communicated by G. E. Wall

\section{Preliminaries}

A map $f: A \rightarrow B$ in a category $\mathscr{C}$ is called monic if $f g=f h$ implies that $g=h$ for all maps $g, h: C \rightarrow A$; it is called epic if $g f=h f$ implies that $g=h$ for all maps $g, h: B \rightarrow C$. An object $A \in \mathscr{C}$ is called an $S$-object if every monic map $f: A \rightarrow A$ is also epic; it is called a $Q$-object if every epic map $f: A \rightarrow A$ is also monic. If $A$ is both an $S$-object and a $Q$-object then $A$ is called an $S Q$-object. In the category of sets the $S Q$-sets are the finite sets. In the category of vector spaces over a field $F$ the $S Q$-spaces are precisely the finite dimensional spaces. In the light of these simple examples, it seems reasonable to view the $S Q$-objects of a category as being of 'finite type'. We shall be chiefly concerned with investigating the $S Q$-objects in certain subcategories of the category of locally compact abelian groups.

If $\mathscr{C}$ and $\mathscr{C}^{*}$ are dual categories then a map $f \in \mathscr{C}$ is epic if and only if $f^{*} \in \mathscr{C}^{*}$ is monic, and $f$ is monic if and only if $f^{*}$ is epic. As a result, we have

Proposition 1. If $\mathscr{C}$ and $\mathscr{C} *$ are dual categories, then $A \in \mathscr{C}$ is an SQ-object if and only if $A^{*} \in \mathscr{C}^{*}$ is an $S Q$-object.

Proposition 2. Suppose $\mathscr{C}$ is an additive category, $A_{1}, A_{2} \in \mathscr{C}$, and $B=A_{1} \oplus$ $A_{2}$. (a) If $B$ is an $S Q$-object then $A_{1}$ and $A_{2}$ are $S Q$-objects. (b) If $A_{1}$ and $A_{2}$ are $S Q$-objects, Hom $\left(A_{1}, A_{2}\right)=0$, and Hom $\left(A_{2}, A_{1}\right)=0$, then $B$ is an SQ-object.

Proof. (a) If $f: A_{1} \rightarrow A_{1}$ then there is $[6$, p. 251] a unique map $g: B \rightarrow B$ satisfying either, and hence both, of the dual conditions

(1) $\pi_{1} g=f \pi_{1}$ and $\pi_{2} g=\pi_{2}$, or

(2) $g l_{1}=l_{1} f$ and $g l_{2}=l_{2}$

(the maps $\pi_{j}$ and $l_{j}$ may be thought of as the canonical projections and injections associated with a direct sum). Suppose that $f$ is monic and that $g h=g k$. Then $f \pi_{1} h=\pi_{1} g h=\pi_{1} g k=f \pi_{1} k$, so $\pi_{1} h=\pi_{1} k$. Also $\pi_{2} h=\pi_{2} g h=\pi_{2} g k=\pi_{2} k$. Thus $\imath_{1} \pi_{1} h=\imath_{1} \pi_{1} k, \imath_{2} \pi_{2} h=\imath_{2} \pi_{2} k$, so $h=\imath_{1} \pi_{1} h+\imath_{2} \pi_{2} h=\imath_{1} \pi_{1} k+\imath_{2} \pi_{2} k=k$, 
and $g$ is monic. Since $B$ is $S Q, g$ is also epic. If $h f=k f$, then $h \pi_{1} g=h f \pi_{1}=k f \pi_{1}$ $=k \pi_{1} g$, and so $h=k$, since $\pi_{1} g$ is epic. Thus $f$ is epic. A similar argument shows that if $f$ is epic then $f$ is monic. Thus $A_{1}$ is an $S Q$-object.

The proof of Proposition 4(b), below, is categorical in essence; it provides a proof of Proposition 2(b) as well.

\section{Locally Compact Abelian Groups}

The category of locally compact abelian groups with continuous homomorphisms will be denoted by $\mathscr{L} \mathscr{C} \mathscr{A}$. The subcategories of compact and discrete abelian groups will be denoted by $\mathscr{C} \mathscr{A}$ and $\mathscr{D} \mathscr{A}$, respectively. By the Pontryagin Duality Theorem the category $\mathscr{L} \mathscr{C} \mathscr{A}$ is self dual, and the categories $\mathscr{C} \mathscr{A}$ and $\mathscr{D} \mathscr{A}$ are dual to one another. Note that a map $f \in \mathscr{L} \mathscr{C} \mathscr{A}$ is monic if and only if it is one-to-one, and epic if and only if it has dense range.

The following groups, with their usual topologies, will enter into the discussion: $Z$ will denote the additive group of integers, $R$ the real numbers, $R_{a} \in \mathscr{D} \mathscr{A}$ the rationals, $S_{\boldsymbol{a}} \in \mathscr{C} \mathscr{A}$ the $a$-adic solenoid (where $\boldsymbol{a}=(2,3,4, \cdots)$, see [5, p. 114]), $\Omega_{p} \in \mathscr{L} \mathscr{C} \mathscr{A}$ the $p$-adic numbers, and $\Delta_{p} \in \mathscr{C} \mathscr{A}$ the $p$-adic integers.

Proposition 3. Suppose $G \in \mathscr{L} \mathscr{C} \mathscr{A}$ is the local direct product of groups $G_{\alpha} \in \mathscr{L} \mathscr{C} \mathscr{A}$ relative to open subgroups $H_{\alpha}$ (see [5, p. 56]), and let $\pi_{\alpha}: G \rightarrow G_{\alpha}$ and $l_{\alpha}: G_{\alpha} \rightarrow G$ denote the canonical projections and injections. Suppose $H \in \mathscr{L} \mathscr{C} \mathscr{A}$. (a) If $g, h \in \operatorname{Hom}(G, H)$ and $g l_{\alpha}=h l_{\alpha}$, all $\alpha$, then $g=h$. (b) If $g, h \in \operatorname{Hom}(H, G)$ and $\pi_{\alpha} g=\pi_{\alpha} h$, all $\alpha$, then $g=h$.

The proof is elementary, and will be omitted.

Proposition 4. Suppose $G \in \mathscr{L} \mathscr{C} \mathscr{A}$ is the local direct product of groups $G_{\alpha} \in \mathscr{L} \mathscr{C} \mathscr{A}$. (a) If $G$ is $S Q$ then each $G_{\alpha}$ is $S Q$. (b) If each $G_{\alpha}$ is $S Q$ and if $\operatorname{Hom}\left(G_{\alpha}, G_{\beta}\right)=0$ for $\alpha \neq \beta$ then $G$ is $S Q$.

PROOF. (a) $\mathscr{L} \mathscr{C} \mathscr{A}$ is an additive category, and $G$ is topologically isomorphic with $G_{\alpha} \oplus G^{\prime}$, where $G^{\prime}$ is the local direct product of all $G_{\beta}, \beta \neq \alpha$. By Proposition 2(a), $G_{\alpha}$ is $S Q$. (b) If $f \in \operatorname{Hom}(G, G)$, define $f_{\alpha}=\pi_{\alpha} f_{\alpha} \in \operatorname{Hom}\left(G_{\alpha}, G_{\alpha}\right)$ for each $\alpha$. We show that $f$ is monic (epic) if and only if every $f_{\alpha}$ is monic (epic). Observe that $\pi_{\alpha} l_{x} \pi_{\alpha}=\pi_{\alpha}$, and hence that $\pi_{\alpha} f l_{\alpha}=\pi_{\alpha} l_{\alpha} \pi_{\alpha} f l_{\alpha}$. Also $\pi_{\beta} f l_{\alpha}=\pi_{\beta} l_{\alpha} \pi_{\alpha} f_{l_{\alpha}}=0$ if $\beta \neq \alpha$, and so, by Proposition 3(b), $f l_{\alpha}=l_{\alpha} \pi_{\alpha} f_{l_{\alpha}}=l_{\alpha} f_{\alpha}$ for all $\alpha$. A similar argument shows that $\pi_{\alpha} f=f_{\alpha} \pi_{\alpha}$ for all $\alpha$.

Suppose then that $f \in \operatorname{Hom}(G, G)$ is monic and that $g, h \in \operatorname{Hom}\left(H, G_{\alpha}\right)$, with $f_{\alpha} g=f_{\alpha} h$. Then $f_{l_{\alpha}} g=\imath_{\alpha} f_{\alpha} g=\iota_{\alpha} f_{\alpha} h=f_{l_{\alpha}} h$. Since $f l_{\alpha}$ is monic we have $g=h$, and so $f_{\alpha}$ is monic.

Suppose every $f_{\alpha}$ is monic and that $g, h \in \operatorname{Hom}(H, G)$, with $f g=f h$. Then $f_{\alpha} \pi_{\alpha} g=\pi_{\alpha} f g=\pi_{\alpha} f h=f_{\alpha} \pi_{\alpha} h$, so $\pi_{\alpha} g=\pi_{\alpha} h$ for all $\alpha$. Thus $g=h$ by Proposition $3(\mathrm{~b})$, and so $f$ is monic. 
An analogous argument establishes that $f$ is epic if and only if each $f_{\alpha}$ is epic, and the proposition follows immediately.

Corollary 1. Suppose $G \in \mathscr{D} \mathscr{A}$ is a torsion group, with p-primary component $G_{p}$ for each prime $p$. Then $G$ is $S Q$ if and only if each $G_{p}$ is $S Q$.

An example of an infinite primary $S Q$-group in $\mathscr{D} \mathscr{A}$ was given by Pierce [8, p. 302]. His construction was simplified by Megibben [7, p. 158]. It has been shown by Beaumont and Pierce [3,pp. 213 and 218] that any infinite primary $S$-group (hence any infinite primary $S Q$-group) must be uncountable but have cardinality less than or equal to that of the continuum.

We give another corollary to Proposition 4 that will prove useful later. If $n(p)$ is a cardinal number then $\Omega_{p}^{n(p)^{\prime}}$ denotes the group consisting of all elements $x \in \Omega_{p}^{n(p)}$ for which the set of values of the $p$-adic valuation of the components of $x$ is bounded. It is shown in [5, p. 420] that $\Omega_{p}^{n(p)^{\prime}} \in \mathscr{L} \mathscr{C} \mathscr{A}$ is an injective envelope (minimal divisible extension) for $\Delta_{p}^{n(p)}$. Furthermore, the local direct product $E$ of the groups $\Omega_{p}^{n(p)^{\prime}}$ relative to the open subgroups $\Delta_{p}^{n(p)}$ is an injective envelope for $\prod_{p} \Delta_{p}^{n(p)}$.

COROLlary 2. The group $E$ (above) is $S Q$ if and only if each $n(p)$ is finite.

Proof. Observe that if $f \in \operatorname{Hom}\left(\Omega_{p}, \Omega_{q}\right)$ then $f(r)=r f(1)$ for every $r \in R_{a}$ (viewing $R_{a}$ as a subfield of both $\Omega_{p}$ and $\Omega_{q}$ ). Since $f$ is continuous and $\Omega_{q}$ is a topological field it follows that every $p$-Cauchy sequence in $R_{a}$ is also a $q$-Cauchy sequence unless $f(1)=0$, in which case $f=0$. But if $p \neq q$ and $a_{n}=\sum_{1}^{n} p^{k}$ then it is easy to see that $\left\{a_{n}\right\}$ is $p$-Cauchy but not $q$-Cauchy. Thus $\operatorname{Hom}\left(\Omega_{p}, \Omega_{q}\right)=0$ if $p \neq q$.

Suppose $E$ is $S Q$. If some $n(p)$ were infinite then clearly a shift map on $\Omega_{p}^{n(p)^{\prime}}$ would be monic but not epic, contradicting Proposition 4(a). Suppose then that every $n(p)$ is finite. If $f \in \operatorname{Hom}\left(\Omega_{p}, \Omega_{p}\right)$, then $f(r)=r f(1)$ for all $r \in R_{a}$, and hence $f(x)=x f(1)$ for all $x \in \Omega_{p}$, since $R_{a}$ is dense in $\Omega_{p}$ and $f$ is continuous. Thus $f$ is either 0 or an isomorphism. If $\Omega_{p}^{n(p)^{\prime}}=\Omega_{p}^{n(p)}$ is viewed as a vector space over $\Omega_{p}$ then clearly every $f \in \operatorname{Hom}\left(\Omega_{p}^{n(p)}, \Omega_{p}^{n(p)}\right)$ is a linear transformation. It follows that $\Omega_{p}^{n(p)}$ is an $S Q$-group, and hence that $E$ is $S Q$, by Proposition $4(\mathrm{~b})$.

THEOREM 1. If $G \in \mathscr{D} \mathscr{A}$ is torsion free then $G$ is $S Q$ if and only if it is isomorphic with $R_{a}^{n}$ for some non-negative $n \in Z$. Dually, if $G \in \mathscr{C} \mathscr{A}$ is connected then $G$ is $S Q$ if and only if it is (topologically) isomorphic with $S_{a}^{n}$ for some non-negative $n \in Z$.

Proof. (see [2, p. 384]). If $G$ is not divisible then $n G$ is a proper subgroup of $G$ for some positive integer $n$. Thus the map $x \rightarrow n x$ is monic but not epic. As a result $G$ is divisible if it is $S Q$, hence it is a vector space over the field $R_{a}$. Every map $f: G \rightarrow G$ is a linear transformation so $G$ is an $S Q$-group if and only if it is finite dimensional as a vector space over $R_{a}$. The dual statement follows from Proposition 1 since $G \in \mathscr{D} \mathscr{A}$ is torsion free if and only if $G^{*} \in \mathscr{C} \mathscr{A}$ is connected, the duality preserves direct sums, and $R_{a}^{*}=S_{a}$ (see [5, pp. 385 and 404]). 
An investigation of (discrete) $S$-groups and $Q$-groups was conducted by R. Baer in [1]. Special cases of two theorems from [1, pp. 268 and 274] can be combined to give a set of sufficient conditions in order that $G$ should be an $S Q$ group.

BAER's TheOrem. Suppose $G \in \mathscr{D} \mathscr{A}$ has a finite chain of subgroups $0=$ $H_{0} \subseteq H_{1} \subseteq \cdots \subseteq H_{n}=G$, satisfying

(1) iff: $G \rightarrow G$ is monic, then $f H_{i} \subseteq H_{i}$,

(2) iff: $G \rightarrow G$ is epic, then $f H_{i}=H_{i}$, and

(3) $H_{i+1} / H_{i}$ is an $S Q$-group, $i=0,1, \cdots, n-1$.

Then $G$ is an $S Q$-group.

The proof, which is valid even for $G$ nonabelian, will not be reproduced here. The essential steps may be seen, however, in the proof of Theorem 3, below, if the topological details are ignored.

THEOREM 2. Suppose $G \in \mathscr{D} \mathscr{A}$ has torsion subgroup $T$. If $T$ and $G / T$ are $S Q$ groups then $G$ is an $S Q$-group. Dually, suppose $G \in \mathscr{C} \mathscr{A}$ has connected component of the identity $C$. If $C$ and $G / C$ are $S Q$-groups then $G$ is an $S Q$-group.

Proof. We prove only the first statement; the dual statement follows as in the proof of Theorem 1.

Set $H_{0}=0, H_{1}=T$, and $H_{2}=G$. If $f: G \rightarrow G$ is epic, set $g(x+T)=f x+T$. Then $g$ is clearly well defined and epic in $\operatorname{Hom}(G / T, G / T)$. Since $G / T$ is $S Q, g$ is also monic, so $f x+T=T$ if and only if $x \in T$, i.e. $f x \in T$ if and only if $x \in T$. Thus $f \mid T$ is epic in Hom $(T, T)$, and condition (2) of Baer's Theorem holds. Conditions (1) and (3) obviously hold, so $G$ is an $S Q$-group.

COROllary. If $G \in \mathscr{D} \mathscr{A}$ splits, i.e. $G \cong T \oplus G / T$, then $G$ is $S Q$ if and only if both $T$ and $G / T$ are $S Q$.

It is reasonable to ask whether the splitting hypothesis in the corollary is necessary. The answer, unfortunately, is yes, and as a result the torsion free and primary cases are not independent of one another in $\mathscr{D} \mathscr{A}$. For example, let $G_{p}$ be the integers $\bmod p$ for each prime $p$ and set $G=\prod_{p} G_{p}$. Then $T=\Sigma_{p} \oplus G_{p}$, and it can be shown that $\operatorname{Hom}(G, G)=\prod_{p} \operatorname{Hom}\left(G_{p}, G_{p}\right)$ in the obvious fashion. It follows easily that $G$ is $S Q$. Also, $T$ is $S Q$ by Proposition 4, Corollary 1 . However, $G / T$ is divisible and infinite dimensional as a vector space over $R_{a}$. Thus, by Theorem $1, G / T$ is not an $S Q$-group.

The next theorem is a topological version of Baer's Theorem.

TheOREM 3. Suppose $G \in \mathscr{L} \mathscr{C} \mathscr{A}$ has a finite chain of subgroups $0=H_{0} \subseteq$ $H_{1} \subseteq \cdots \subseteq H_{n}=G$, satisfying, for $i=0,1, \cdots, n-1$,

(1) if $f: G \rightarrow G$ is monic, then $f H_{i} \subseteq H_{i}$,

(2) if $f: G \rightarrow G$ is epic, then $f H_{i}=H_{i}$, 
(3) $H_{i} \in \mathscr{C} \mathscr{A}$, and

(4) $H_{i+1} / H_{i}$ is $S Q$.

Then $G$ is an $S Q$-group.

ProOF. We prove the theorem with $n=2$, writing $H_{1}=H$. An easy induction completes the proof.

Suppose first that $f: G \rightarrow G$ is monic. Then $f \mid H$ is epic, since $H$ is $S Q$. If $q: G \rightarrow G / H$ is the quotient map then $q f$ induces a homomorphism $g: G / H \rightarrow G / H$ via $g(x+H)=f x+H$, i.e. $g q=q f$. If $U$ is an open neighborhood of 0 in $G / H$, set $W=q f^{-1}\left(q^{-1}(U)\right)$. Then $W$ is open in $G / H$ since $f$ is continuous and $q$ is both open and continuous. But $g W=q f f^{-1}\left(q^{-1}(U)\right) \subseteq U$, so $g$ is continuous. Also, ker $q f=\{x: f x \in H\}=H$ since $f$ is monic and $f \mid H$ is epic, and so $g$ is monic. But then $g$ is epic since $G / H$ is $S Q$. Since $q$ is an open map, $q\left(G \backslash f(G)^{-}\right)$is an open subset of $G / H$. If it were not disjoint from $q f G=$ range $g$, then there would exist $x \in G \backslash f(G)^{-}$and $y \in G$ such that $q x=q f y$, i.e. $x-f y \in H$. But $f H=H$, so then $x-f y=f z$ for some $z \in H$. This is impossible because $x \notin f G$. Since $g$ is epic we conclude that $q\left(G \backslash f(G)^{-}\right)$is empty, hence that $f(G)^{-}=G$, i.e. that $f$ is epic.

Suppose next that $f: G \rightarrow G$ is epic, and denote $\operatorname{ker} f$ by $N$. As above, $q f$ induces $g \in \operatorname{Hom}(G / H, G / H)$, with $g q=q f$. Since $H$ is compact $q$ is a closed map [5, p. 37]. Since $f$ is epic we have $G / H=q G=q\left(f(G)^{-}\right)=(q f(G))^{-}=(g q(G))^{-}$ $=(g(G / H))^{-}$, i.e. $g$ is epic. Thus $g$ is monic since $G / H$ is $S Q$, and so $f x+H=$ $g(x+H)=H$ if and only if $x \in H$. In particular, if $x \in N$ then $f x=0$, so $x \in H$, and $N \subseteq H$. But $f \mid H$ is epic and $H$ is $S Q$, so $f \mid H$ is monic and $G$ is an $S Q$-group.

Theorem 4. If $H \in \mathscr{C} \mathscr{A}$ is $S Q$ and $0 \leqq n \in Z$ then $G=R^{n} \oplus H$ is $S Q$ in $\mathscr{L} \mathscr{C} \mathscr{A}$.

Proof. Since maps are continuous in $\mathscr{L} \mathscr{C} \mathscr{A}$ every $f \in \operatorname{Hom}\left(R^{n}, R^{n}\right)$ is a linear transformation if $R^{n}$ is considered as a vector space over $R$, and so $R^{n}$ is $S Q$. If $f \in \operatorname{Hom}(G, G)$ then, since $\operatorname{Hom}\left(H, R^{n}\right)=0$, we have $f(x, y)=(g x$, $h x+k y)$, where $g \in \operatorname{Hom}\left(R^{n}, R^{n}\right), h \in \operatorname{Hom}\left(R^{n}, H\right)$, and $k \in \operatorname{Hom}(H, H)$.

If $f$ is epic then $g$ must clearly be surjective since it is a linear transformation and since the range of $f$ is a subset of (range $g$ ) $\times H$. But then $g$ is also monic, and in fact a homeomorphism. Let us show that $k H=H$. If not, $H \backslash k H$ is an open subset of $H$. Choose $y \in H \backslash k H$ and an open neighborhood $N$ of 0 in $H$ such that $(y+N) \cap(k H+N)$ is empty. Next choose a neighborhood $W$ of 0 in $R^{n}$ such that $h x \in N$ if $x \in W$, and finally choose an open neighborhood $U$ of 0 in $R^{n}$ such that $x \in W$ if $g x \in U\left(g^{-1}\right.$ is continuous). Then $U \times(y+N)$ is an open set in $G$; we show it to be disjoint from the range of $f$. Suppose $(u, v) \in U \times(y+N)$. If also $(u, v) \in$ range $f$, then there exists $(r, s) \in G$ such that $g r=u$ and $h r+k s=v$. But then $r \in W$ and so $h r \in N$. Thus $v=k s+h r \in k H+\mathrm{N}$, contradicting $v \in y+N$.

If we consider $H$ as a subgroup of $G$ then we have just shown that $f H=H$ for every epic $f$ in Hom $(G, G)$. The theorem now follows from Theorem 3 . 
Corollary 1. Suppose $G \in \mathscr{L} \mathscr{C} \mathscr{A}$ is compactly generated. Then $G$ is $S Q$ if and only if it is topologically isomorphic with $R^{n} \oplus H$, where $H \in \mathscr{C} \mathscr{A}$ is $S Q$ and $0 \leqq n \in Z$.

ProOF. Since $G$ is compactly generated it is topologically isomorphic with $R^{n} \oplus Z^{m} \oplus H[5$, p. 90]. If $G$ is $S Q$ then it follows from Proposition 2 that $m=0$ and $H$ is $S Q$.

Corollary 1 , together with Proposition 1, shows that the problem of determining all compactly generated $S Q$-groups in $\mathscr{L} \mathscr{C} \mathscr{A}$ is equivalent with that of determining all $S Q$-groups in the category $\mathscr{D} \mathscr{A}$. An application of Theorem 1 determines all compactly generated connected $S Q$-groups in $\mathscr{L} \mathscr{C} \mathscr{A}$.

CoRollary 2. If $G$ in $\mathscr{L} \mathscr{C} \mathscr{A}$ is compactly generated and connected, then $G$ is $S Q$ if and only if it is topologically isomorphic with $R^{n} \oplus S_{a}^{m}, 0 \leqq n, m \in Z$.

Finally, we determine all divisible torsion free $S Q$-groups in $\mathscr{L} \mathscr{C} \mathscr{A}$.

THEOREM 5. Suppose $G$ in $\mathscr{L} \mathscr{C} \mathscr{A}$ is torsion free and divisible. Then $G$ is $S Q$ if and only if it is topologically isomorphic with $R^{j} \oplus R_{a}^{k} \oplus S_{a}^{m} \oplus E$, where $j, k$, and $m$ are non-negative integers and $E$ is the injective envelope of $\prod_{p} \Delta_{p}^{n(p)}$ with $0 \leqq n(p) \in Z$ for each prime $p$.

Proof. By [5, p. 421] $G$ is topologically isomorphic with $R^{j} \oplus\left(\Sigma \oplus R_{a}\right) \oplus$ $S_{a}^{m} \oplus E$, where $0 \leqq j \in Z, k$ and $m$ are cardinals, there are $k$ copies of $R_{a}$ in the (discrete) direct sum, and $E$ is the injective envelope of $\prod_{p} \Delta_{p}^{n(p)}$, with $n(p)$ a cardinal for each prime $p$. By Proposition 1, Theorem 1, and Corollary 2 of Proposition 4 we see that $m, k$, and all $n(p)$ are finite.

For the converse we first observe that $E$ is self dual. This follows from remarks on page 422 of [5] and the fact that $\Delta_{p}^{\perp} \cong\left(\Omega_{p} / \Delta_{p}\right)^{*} \cong\left(Z\left(p^{\infty}\right)\right)^{*}=\Delta_{p}$. For each prime $p$ let $\pi_{p}$ be the projection on $E$ to $\Omega_{p}^{n(p)}$. If $f \in \operatorname{Hom}\left(R^{j}, E\right)$ then $\pi_{p} f \in$ Hom $\left(R^{j}, \Omega_{p}^{n(p)}\right)$ so $\pi_{p} f=0$ since $R^{j}$ is connected and $\Omega_{p}^{n(p)}$ is totally disconnected. Thus $f=0$ by Proposition 3 , and Hom $\left(R^{j}, E\right)=0$. It follows, since both $R^{j}$ and $E$ are self dual, that $\operatorname{Hom}\left(E, R^{j}\right)=0$. By Proposition 2(b), $R^{j} \oplus E$ is $S Q$. Since $S_{a}^{m}$ is compact and connected we have $\operatorname{Hom}\left(S_{a}^{m}, R^{j} \oplus E\right) \cong \operatorname{Hom}\left(S_{a}^{m}, R^{j}\right) \oplus$ Hom $\left(S_{a}^{m}, E\right)=0$. Viewing $S_{a}^{m}$ as a subgroup of $R^{j} \oplus E \oplus S_{a}^{m}$ we see that $R^{j} \oplus E \oplus S_{a}^{m}$ is $S Q$, by Theorem 3, and hence that $R^{j} \oplus R_{a}^{k} \oplus E$ is $S Q$, substituting $k$ for $m$ and applying Proposition 1. Finally, Hom $\left(S_{a}^{m}, R_{a}^{k}\right)=0$ since $S_{a}$ is connected and $R_{a}$ is discrete, and so Hom $\left(S_{a}^{m}, R^{j} \oplus R_{a}^{k} \oplus E\right)=0$. Another application of Theorem 3 yields the fact that $R^{j} \oplus R_{a}^{k} \oplus S_{a}^{m} \oplus E$ is $S Q$, proving the theorem.

The divisibility hypothesis in Theorem 5 may not be necessary. The argument used in the proof of Theorem 1 yields only denseness of $n G$ in $G$, but not divisibility. 


\section{Remarks}

It might be of interest to study the $S Q$-objects in other specific categories. To mention one example, suppose $\mathscr{B}$ is the category of commutative $B^{*}$-algebras with identity and symmetric algebra homomorphisms. Then $\mathscr{B}$ is dual to the category $\mathscr{T}$ of compact Hausdorff spaces. It can be checked that epic means onto and monic means one-to-one in both categories. Thus if $A \in \mathscr{B}, X \in \mathscr{T}$, and $A=X^{*}=C(X)$, then $A$ is an $S Q$-algebra if and only if $X$ is an $S Q$-space.

An example has been constructed (see [4]) of an infinite compact connected Hausdorff space $X$ for which the only continuous maps $f: X \rightarrow X$ are the identity and constant maps onto single points. Thus $X$ is $S Q$ in $\mathscr{T}$ and $A=C(X)$ is $S Q$ in $\mathscr{B}$.

The first-named author wishes to acknowledge a Summer Research award from the University of Oregon Office of Scientific and Scholarly Research. The second-named author acknowledges the generous hospitality of Yale University while he was on a research leave from Syracuse University.

\section{References}

[1] R. Baer, 'Groups without proper isomorphic quotient groups', Bull. Amer. Math. Soc. 50 (1944), 267-278.

[2] R. Beaumont, 'Groups with proper isomorphic subgroups', Bull. Amer. Math. Soc. 51 (1945), $381-387$.

[3] R. Beaumont and R. Pierce, 'Partly transitive modules and modules with proper isomorphic submodules', Trans. Amer. Math. Soc. 91 (1959), 209-219.

[4] H. Cook, 'Continua which admit only the identity mapping onto nondegenerate subcontinua', Fundamenta Math. 60 (1967), 241-249.

[5] E. Hewitt and K. Ross, Abstract Harmonic Analysis, I (Springer, New York, 1963).

[6] S. MacLane, Homology (Springer, Berlin, 1963).

[7] C. Megibben, 'Large subgroups and small homomorphisms', Michigan Math. J. 13 (1966), 153-160.

[8] R. Pierce, 'Homomorphisms of primary abelian groups' in Topics in Abelian Groups, (Scott, Foresman, Chicago, 1963).

The University of Oregon

Syracuse University 